\title{
Synthesis of SiC Based Fibers with Continuous Pore Structure by Melt- spinning and Controlled Curing Method
}

\author{
Ken'ichiro Kita ${ }^{1, a}$, Masaki Narisawa ${ }^{1, b}$, Hiroshi Mabuchi ${ }^{1, c}$, Masayoshi $^{1}$ Itoh $^{2, \mathrm{~d}}$ \\ Masaki Sugimoto $^{3, \mathrm{e}}$, and Masahito Yoshikawa ${ }^{3, \mathrm{f}}$ \\ ${ }^{1}$ Graduate School of engineering, Osaka Prefecture University, 1-1 Gakuen-Cho, Naka-Ku, Sakai, \\ Osaka, 599-8531, Japan \\ ${ }^{2}$ Department of Chemistry and Biochemistry, Fukushima National College of Technology, Taira, \\ Iwaki, Fukushima 970-8034, Japan \\ ${ }^{3}$ Quantum Beam Science Directorate, Japan Atomic Energy Agency, Takasaki, Gunma, 370-1292, \\ Japan \\ ahz302@mtr.osakafu-u.ac.jp, ${ }^{b}$ nar@mtr.osakafu-u.ac.jp, ${ }^{c}$ mabuchi@mtr.osakafu-u.ac.jp, \\ dm-itoh@fukushima-nct.ac.jp, ${ }^{\mathrm{e}}$ sugimoto.masaki@jaea.go.jp, foshikawa.masahito@jaea.go.jp
}

Keywords: Silicon carbide, polymer precursor method, micro tube, ceramic fiber, polycarbosilane, polysiloxane.

\begin{abstract}
Silicon carbide ( $\mathrm{SiC}$ ) based fibers with continuous pore structures were synthesized by the precursor method using a polycarbosilane (PCS) and polymethylhydrosiloxane (PMHS) polymer blends. The pore formation process can be explained by hydrogen gas dissolution in the polymer melt and desaturation process of the dissolved gas during the fiber spinning. We investigated the effect of PMHS additives with different chemical and physical natures on the obtained pore structures, because PMHS decomposition process played a role of hydrogen gas source. The individual polymer melts were characterized by viscosity measurement, gas chromatograph analysis and thermogravimetric (TG) analysis in order to obtain details of pore structure control.
\end{abstract}

\section{Introduction}

The SiC-based continuous fibers, which are mainly used as reinforcements in ceramic matrix composites (CMC) [1-3], are synthesized from polycarbosilane (PCS) through melt-spinning, curing and pyrolysis. This process was invented by Yajima's in 1970s and called "the precursor method" [4]. After this invention, many studies have been presented for the fiber characterization, the advanced fiber synthesis processes and the applications for CMC [5,6].

In recent years, we have investigated compatibilities of various siloxane polymers for PCS in order to synthesis fine fibers, and accidentally found hollow structures at the fiber center in the case of polymethylhydrosiloxane (PMHS) addition [7]. Such central pore formation process can be explained by hydrogen gas dissolution in the polymer melt and desaturation process during the fiber spinning.

In this study, we report the influence of kinds of the additive PMHS on the formed pore structures in the fibers, because chemical and physical properties of polysiloxanes are various in general because of differences in molecular structure (molecular weight, branching and terminal groups). Such knowledge about polymer blend melts contributes pore structure controls in synthesized Si-C-O fibers.

\section{Experiment Procedure}

Two kinds of PMHS (KF-99 and KF-9901, Shin-etsu Chemicals Co., Ltd., Japan) with different chemical and physical natures having $\mathrm{Si}-\mathrm{H}$ groups were prepared for additives of fiber spinning. FT-IR spectra and thermogravimetric (TG) curves of PMHSs were investigated to establish the difference. After the analysis, each PMHSs was blended with PCS (NIPUSI Type-A, Nippon Carbon, 
Japan). The polymer blend including KF-99 was identified as HS15 and the polymer blend including KF-9901 was identified as HSa15. Viscosities and TG curve measurements were performed on each polymer blends in heating at 523-613K. The decomposition gas evolved from the samples by heat treatment up to $573 \mathrm{~K}$ was analyzed by gas chromatograph.

After these characterizations, each polymer blends were melt-spun to fibers around $573 \mathrm{~K}$ and cured by thermal oxidation, $\gamma$-ray oxidation curing or electron beam curing. The heating rate for the thermal curing was $8 \mathrm{~K} / \mathrm{h}$ up to $458 \mathrm{~K}$ under air flow. $\gamma$-ray curing under air was performed with the dose rate of $3.31 \mathrm{~Gy} / \mathrm{s}$ and the total dose was $1.14 \mathrm{MGy}$. Electron beam curing under inert atmosphere was performed with the dose rate of $0.4-1.58 \mathrm{kGy} / \mathrm{s}$ and the total dose in this case was about $10 \mathrm{MGy}$. The remained radicals were annealed at $773 \mathrm{~K}$.

The fibers after some curing were pyrolized at $1273 \mathrm{~K}$ and observed by FE-SEM. After the pyrolysis at $1273 \mathrm{~K}$, the cross-sections of these fibers were also observed by FE-SEM.

\section{Results}

Figure 1 shows IR spectra of KF-99 and KF-9901. Each spectrum was normalized by $\mathrm{Si}-\mathrm{CH}_{3}$ absorption bands at $1250 \mathrm{~cm}^{-1}$. The Si-O-Si absorption band at 1050-1250 $\mathrm{cm}^{-1}$ in spectrum of KF-9901 is observed at lower wave number than that of KF-99. On the other hand, the $\mathrm{Si}-\mathrm{CH}_{3}$ absorption bands at $850 \mathrm{~cm}^{-1}$ in $\mathrm{KF}-9901$ is stronger than that in KF-99.

Figure 2 shows TG curves of KF-99 and KF-9901 in heating up to $573 \mathrm{~K}$. Beyond $553 \mathrm{~K}$, the mass of KF-99 apparently decreases. On the other hand, the mass of KF-9901 constantly decreases. The residual mass at $573 \mathrm{~K}$ is $44.5 \%$ for KF-99 or $80.0 \%$ for KF-9901. KF-9901 apparently shows higher thermal stability than KF-99. Figure 3 shows viscosities of PCS, HS15 and HSa15 at $533-573 \mathrm{~K}$. The viscosity of PCS starts to decrease at $573 \mathrm{~K}$ and that of HS15 starts to decrease at $513 \mathrm{~K}$ and that of HSa15 starts to decrease at $533 \mathrm{~K}$. In the same temperature, the viscosity of HSa15 was always higher than that of HS15. Molecular weight of KF-9901 is possibly larger than that of KF-99.

Table 1 shows Gas Chromatograph (GC) analysis data on accumulated decomposition gases from HS15 and HSa15 up to $573 \mathrm{~K}$. The total amount of evolved gas from HS15 or HS15a was $2.13 \times 10^{-4} \mathrm{~g} / \mathrm{mol}$, or $1.5 \times 10^{-4} \mathrm{~g} / \mathrm{mol}$, respectively. The difference in total evolved gas amount is explained by the difference in hydrogen gas evolution, because hydrogen is major component in the evolved gas. From the above results, it is considered that $\mathrm{H}$-oil plays 2 roles of a plasticizer and a blowing agent of hydrogen.

Figure 4 shows the SEM images of the cross sections of HS15 and HSa15 fibers after pyrolysis at $1273 \mathrm{~K}$. The most of cross sections of HS15 fibers include single hollow (Fig.4-(a),(b)). In some cross section, multiple pores

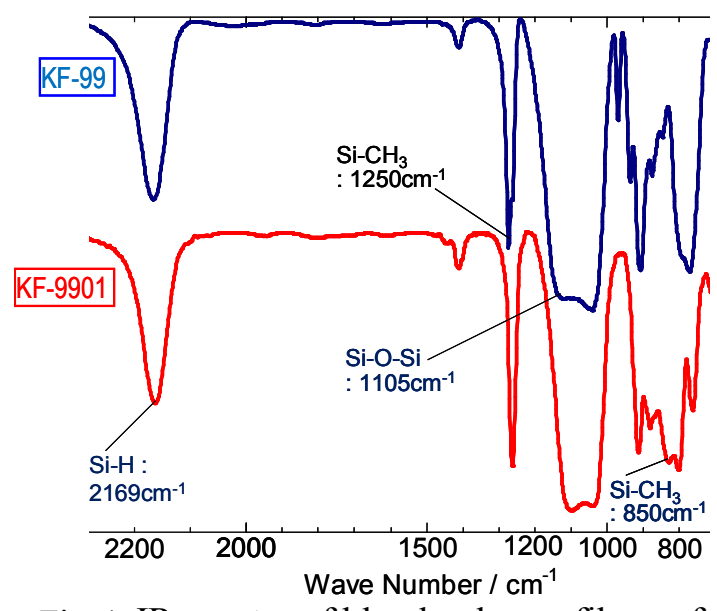

Fig. 1 IR spectra of blend polymer fibers of KF-99 and KF-9901.

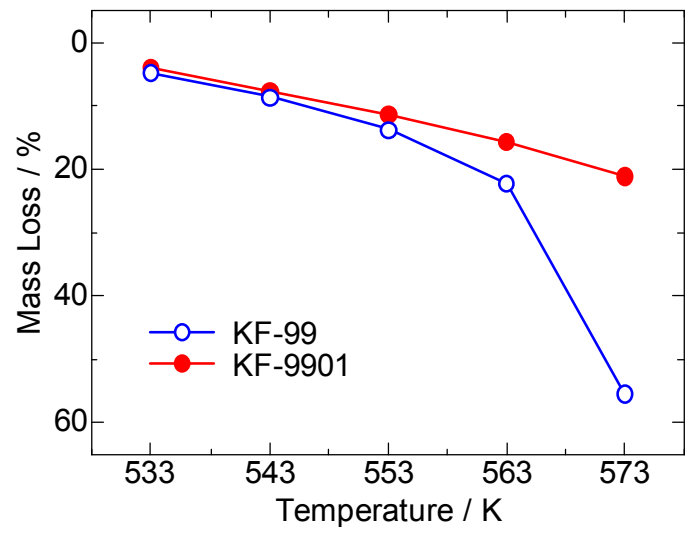

Fig. 2 TG curves of KF-99 and KF-9901 in heating at $533 \mathrm{~K}$ up to $573 \mathrm{~K}$.

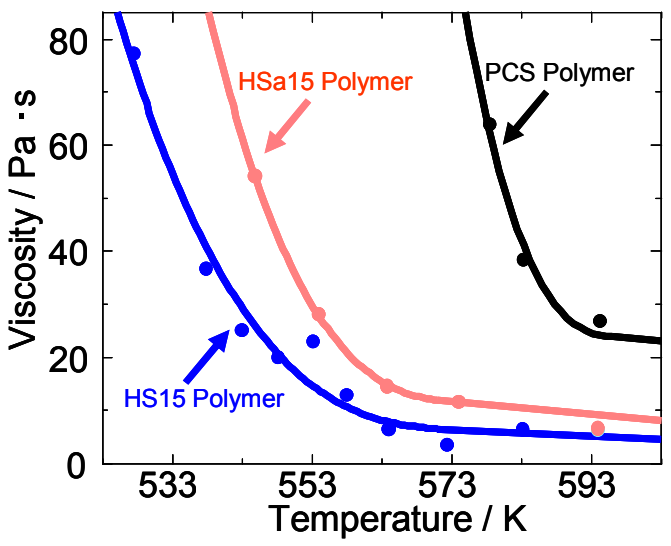

Fig. 3 Viscosities of PCS and polymer blend (HS15, HSa15) in heating up to $603 \mathrm{~K}$. 
structure (single large pore with two or three satellite pores) is observed. On the other hand, the cross section of HSa15 fibers include a number of sub-micro sized pores (Fig.4-(c),(d)).

In melt-spinning, the pressure in the spinneret and the temperature of the polymer is rapidly decreased at the same time and the dissolved gas leaves from the polymers. Moreover, this process will proceed from the outer shell of the as-spun fiber, and the desaturated gas will be formed along the fiber of the longitudinal axis [8]. In the same temperature, the viscosity of HS15 is low and the large amount of hydrogen gas possibly dissolves in the melt as compared with HSa15. In such situation, pore formation and pore coarsening process in HS15 is accelerated, and a single pore structure is formed during melt spinning. On the other hand, the high viscosity of HSa15 melt prevents pore coarsening, and tiny pores are formed during melt-spinning. This process using HSal5 is promising to make porous fibers.

The mass gain of the HS15 fibers after thermal oxidation curing was 5.1\% and that of HS 15 fibers after $\gamma$-ray oxidation curing was $14.2 \%$. These mass gains correspond to oxygen capturing in the fibers. In thermal oxidation curing, oxygen in the air flow was mainly captured at the vicinity of the surface of the fibers. In $\gamma$-ray oxidation curing, free radicals were formed in a whole fiber at room temperature. In such cases oxygen possibly penetrates into fiber core without forming skin layer. The mass gain of HS15 fiber after electron beam curing was almost 0 and this result is natural because curing process proceeds with radical annealing at $773 \mathrm{~K}$ without oxygen. The ceramics yield after pyrolysis of HS15 fibers with thermal oxidation curing, $\gamma$-ray oxidation curing or electron beam curing without oxygen was about $88.6,93.3$, or $82.0 \%$, respectively. These ceramics yield roughly corresponds to quantity of oxygen captured by the fiber.

Figure 5 shows the SEM images of HS15 fibers pyrolized at $1273 \mathrm{~K}$ after various curing method ((a): Thermal oxidation curing, (b): $\gamma$-ray oxidation curing, (c): Electron beam curing without oxidation). In the pyrolysis at $1273 \mathrm{~K}$, there was no difference in the appearance among these fibers; the cross sections of all these fibers were not porous except the single central pore, the inside surface of the central pore was smooth, and the outside surface of these fibers were slightly wrinkled.
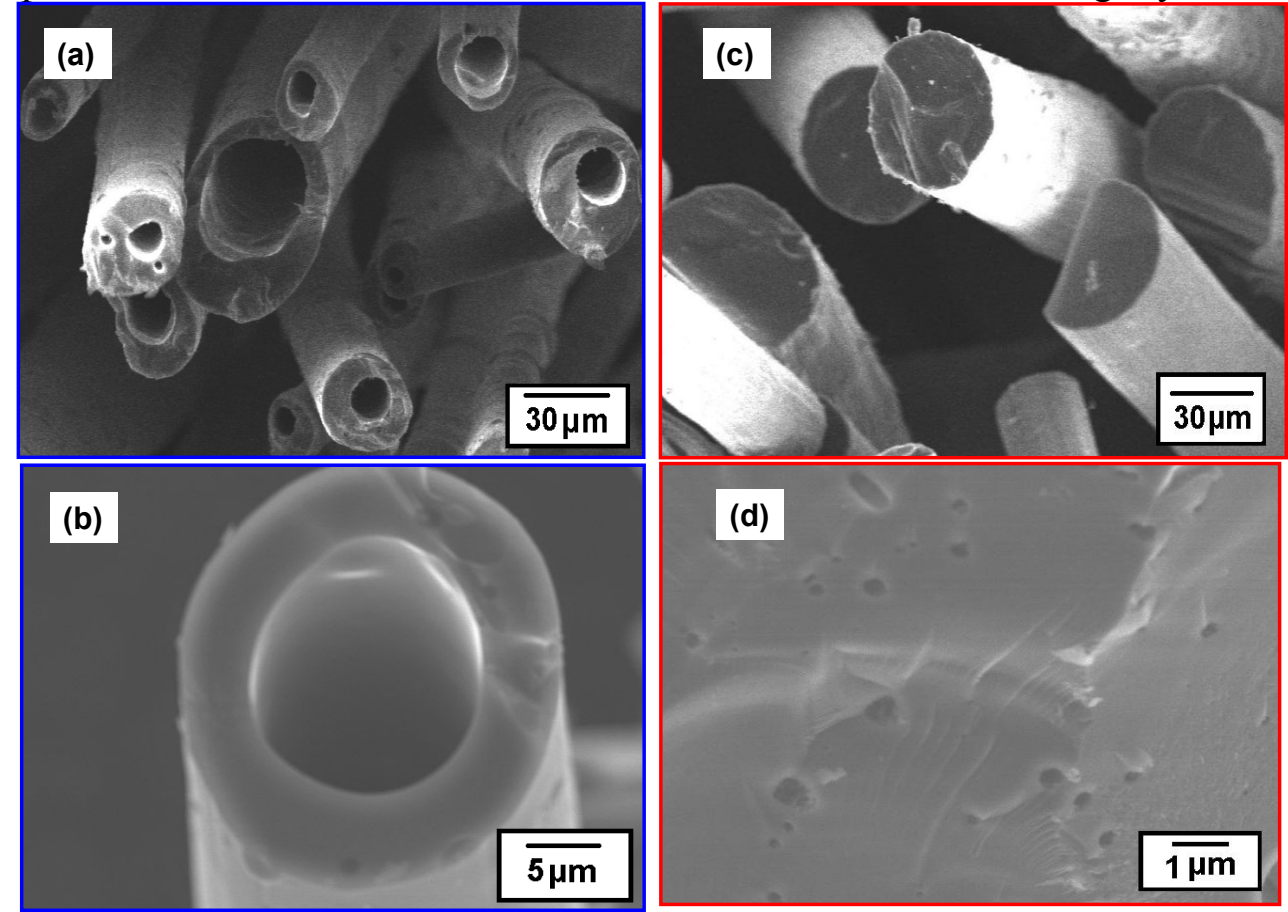

Fig. 4 FE-SEM graphs of the cross sections of HS15 and HSa15 Fibers after thermal oxidation curing and pyrolysis at $1273 \mathrm{~K}$. ((a)(b):HS15 fibers, (c)(d):HSa15 fibers) 

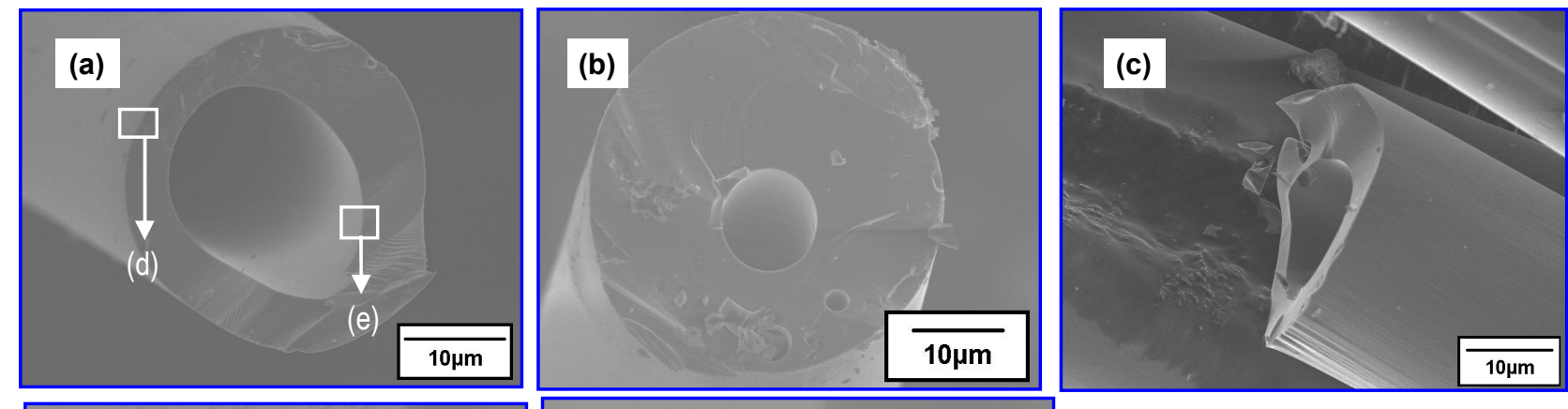

(d)

(e)

(a) : thermal oxidation cured fiber

(b) : $\gamma$-ray oxidation cured fiber

(c) : fiber cured by electron beam without oxidation

(d) : outer surface of thermal oxidation cured fiber

(e) : inner wall of thermal oxidation cured fiber

$1 \overline{100 \mathrm{~nm}}$ oxidation cured fiber

Fig. 5 FE-SEM graphs of the cross sections of HS15 fibers after pyrolysis at $1273 \mathrm{~K}$ by thermal oxidation curing, $\gamma$-ray oxidation curing and electron beam curing without oxidation.

\section{Summary}

The effect of PHMS additives to PCS on pore formation in melt spun fiber is analyzed from the viewpoint of melt viscosity and an amount of evolved gas from the polymer melt. KF-99 additive with low molecular weight, which evolves a larger amount of hydrogen during the heating and possesses higher efficiency to soften the polymer melt, yields the single pore Si-C-O fiber after pyrolysis. KF-9901 additive with high molecular weight, which evolves a smaller amount of hydrogen during the heating and possesses lower efficiency to soften the polymer melt, yields the porous Si-C-O fibers with sub-micro pores after pyrolysis. The ceramics yield of the HS15 fibers is sensitive to the adopted curing process (thermal, $\gamma$-ray or electron beam) before pyrolysis at $1273 \mathrm{~K}$. All the fibers keep hollow structure after pyrolysis at $1273 \mathrm{~K}$, although the $\mathrm{Si}-\mathrm{C}-\mathrm{O}$ chemical composition is various.

\section{Acknowledgements}

A part of this work was supported by Grant-in Aid for Scientific Research C from the Japan Society of Promotion Science and the cooperative research program of Inter-University Laboratory for the Common Use of Nuclear Facilities from The University of Tokyo.

\section{References}

[1] K. Okamura: Composites Vol. 18 (1987), p. 107

[2] T. Yamamura, T. Ishikawa, M. Shibuya, T. Hisayuki and K. Okamura: J. Mater. Sci. Vol. 23 (1988), p. 2589

[3] R. Naslain: Compos. Sci. Tech. Vol. 64 (2004), p. 155

[4] S. Yajima, K. Okamura, J. Hayashi, M. Omori: J. Am. Ceram. Soc. Vol. 59 (1976), p. 324

[5] K. Okamura and T. Seguchi: J. Inorg. Organomet. Polym. Vol.2 (1992), p. 171

[6] K. Kumagawa, H. Yamaoka, M. Shibuya and T. Yamamura: Ceram. Eng. Sci. Pro. (1997), p.987

[7] K. Kita, M. Narisawa, H. Mabuchi and M. Itoh: Key Eng. Mater. Vol. 352 (2007), p. 69

[8] H. Nakajima, T. Ikeda and S.K. Hyun: Adv. Eng. Mater. Vol.6 (2004), p. 377 